\title{
Contributing Ad Hoc Reviewers 2021
}

Rehabilitation Psychology publishes articles that capture the breadth of science and practice in rehabilitation psychology. To achieve this mission, we rely on a diverse group of individuals who serve as ad hoc reviewers. We are grateful to the following individuals for their generous commitment of time and effort for the 2021 volume of Rehabilitation Psychology. If you would like to join this group of individuals by serving as an ad hoc reviewer, please contact the editor at ehde@uw.edu.

Rachel Aaron
Leah Adams
Stephanie Danielle Agtarap
Ted Allaire
Elisabet Alzueta
Herb Ames
Anne Arewasikporn
James Arruda
Caitlyn Aruitiunov*
Kara Ayers
Dana Bakula
Anna Banik
Katherine Baum
Kathleen T. Bechtold
Jagriti 'Jackie' Bhattarai
John Parle Blake
Britton Brewer
Patrick Brice
Kathleen Brown
Maggi A. Budd
Lester Butt
Caitlin Campbell
Fong Chan
Chetwyn Chan
Yen Chen
Yen T. Chen*
Joyce Chung
Kathleen Chwalisz
Nancy Ciccollela
Sarah W. Clark
Ashley N. Clausen
Laura Coffey
Matthew L. Cohen
Kristin Jane Conover
Andrew Cook
Bridget Cotner
Ashley Craig
Laura Crocker
Roxana Delgado

*Denotes Co-reviewers.
Thomas Dirth

Ewa Domagała-Zyśk

Charles Drebing

Laura Dreer

Mary Driscoll

Erin Edwards*

Adva Eichengreen

Bella Etingen

Heather A. Feldner

Jacob A. Finn

Martin Forchheimer

Emily Foxen-Craft

Carli Friedman

Nora Fritz

Jérôme Gauvin-Lepage

Chantea D. Goetz

Jessica Graham

Elizabeth S. Gromisch

Huseyin Gulec

Jenni Hannin

Abigail Hardin

Debra A. Harley

Erica Healey

Richard Henry

Tracy Herring

Caroline Hinkle*

Shivayogi V. Hiremath

Grayson Holmbeck

Amy Jak

Marissa Jaross*

Erica K. Johnson

Brian Johnson

Kate Jones

Tsubasa Kawasaki

Quinn Kellerman

Jason E. Kisser

\section{Jaclyn Klepper* \\ Lindsey Knowles \\ Victoria Kordovski* \\ Denise Krch \\ Tracy Kretzmer \\ Angela Kuemmel \\ Hannah Kuper \\ Merve Kurt \\ Duygu Kuzu}

Sarah Lahey

Flavia Lecciso

Jaylene Lee*

Dan Leibel*

Irene W. Leigh

Anthony H. Lequerica

Allison Levine

Junfei Lu

Gloria Yuet Kwan Ma

Sara Hoffman Marchese

Michael F. Martelli

Michele McDonnall

Jed N. McGiffin

Grace McKee

Melody Mickens

Linda R. Mona

Donna Morere

Jeri Morris

Sara Mulroy

Johanna Nielsen*

Amber O'Shea

Deirdre O'Sullivan

Jaclyn Lennon Papadakis

Roxanna Nasseri Pebdani

Angie Philippus*

Kala Phillips

Lawrence Pick

Bradford S. Pierce
Demietrice Pittman

David E. Reed

Elizabeth J. Richardson

Michelle Roberts

Ed Rohn

David Rothman

Jared Rowland

Katherine S. Salamon

Marcia J. Scherer

Katlin Schultz*

Eric Scott

Haley Scott

Robert Shura

Noah D. Silverberg

Arielle Silverman

Beth Springate

Amy Starosta

William Stiers

Kirk J. Stucky

Megan Sutter

Alicia A. Swan

Meredith Jan Tackett

Lauren Terhorst

Salom Teshale

Alice Theadom

Emre Umucu

Ashley Vaillancourt

Thomas Valentine

Dustin Wallace

Katie Wang

Erica Weber

Daniel Whibley

Jennifer Wilson

Catherine Wilson

Adrien Winning*

Ye Zhang 